\title{
LIV.
}

\section{Ueber eine analytische Eigenschaft der geistigen und Milchsäure-Gährung und ihre Anwendung zur Kenntniss der 'Zuckerarten.}

Von

\section{Dubrunfaut.}

(Ann. de Chim. et de Phys. T. XXI, p. 169.)

Schon seit dem Jabre 1830 erkannte ich, dass der Rohrzucker, ehe er die geistige Gährung eingehen kann, eine Umwandlung erleidet, durch welche er dem Traubenzucher ähnlich wird. Die Richtigkeit dieser Beobachtung wurde seitdem durch mehrere Gelehrte und hauptsächlich durch die von Biot entdeckte optische Reaction bestäligt.

Die Untersuchung der geistigen Gährung durch optische Beobachtungen wurde von Biot selbst begonnen und von Mitscherlich und Soubeiran fortgesetzt; die Untersuchungen dieser Gelehrten beschränkten sich aber darauf, nachzuweisen, dass die anderen gährungsfähigen Zuckerarten, als der Rohrzucker, während der Gährung ihre ursprüngliche Drehungsrichtung nicht verändern.

Bei sorgfältiger Wiederholung dieser Versuche bam ich zu folgenden Resultaten:

Wenn man eine Lösung von umgewandeltem Zucker (sucre interverti), dessen Rotation man bei einer Temperatur von $13^{\circ}$ gemessen hat, gähren lässt, so verăndert sich die Rotation der Flüssigkeit so lange nicht, als ${ }_{5}^{3}$ ungefähr des Gewichts an Zucker nicht in Alkohol übergegangen sind. Dieser Versuch wird leicht ausgelührt, indem man die gährende Flüssigkeit theilt und die Theile zu gleicher Zeit den optischen Beobachtungen und der Destillation unterwirft, um den Gang der Alkoholerzeugung mit den Veränderungen im Drehungsvermögen messen zu können.

Von diesem Zeitpuncte an, d. h. nachdem ungefähr $\frac{3}{5}$ des dem Versuche unterworfenen Zuckers in Alkohol umgewandelt sind, bemerkt man, dass die Drehung in ibrer Intensität Ver- 
änderungen erleidet. Vergleicht man diese Veränderungen, die sich durch die Sexagesimalgrade des Bio t'schen Apparates ausdrücken lassen, mit der zugleich erzeugten und mit dem Gay-Lussac'schen Alkoholometer gemessenen Menge Alkohol, so findet man, dass, wenn sich der Alkohol in arithmetischem Verhältnisse entwickelt, die Abnahme der Abweichungsgrade in geometrischer Progression erfolgt.

Diese einfache optische Analyse der geistigen Gährung des umgewandelten Zuckers genügt zu der Annahme, dass dieser Zucker kein einfacher sei, sondern aus verschiedenartigen Elementen mit entgegengesetzter Drehung bestehe. Diese Annahme wird übrigens durch rein chemische Versuche bestätigt, die ich weiter unten angreben werde.

Um Weitläufigkeiten $z u$ vermeiden, gebe ich dieser eigenthūmlichen Art der so eben beschriebenen geistigen Gährung den Namen: Elective geistige Gührung (fermentation alcoholique éleclive).

Stellt man einen ähnlichen Versuch mit Zuckerarten an, welche, wie der reine Traubenzucker, chemisch einfach zu sein scheinen, so bemerkt man zu allen Zeiten der Gährung, dass die Verminderung der Abweichungsgrade der in derselben Zeit erzeugten Alkoholmenge proportional ist. Hierbei ist ebenfalls die optische Reaction in völligem Einklange mit den rein chemischen Methoden, um die einfache Natur des dem Versuche unterworfenen Körpers festzustellen.

Vermischt man absichtlich einfache Zuckerarten und beobachtet in diesen Gemengen das Fortschreiten der geistigen Gährung und die Abnahme der Drehung, so bemerkt man keine durch diese Reaction bewirkte Analyse, d. h. zu allen Zeiträumen der Gährung entsprechen gleiche Mengen erzengten Alkohols gleichen Drehungsabnahmen. Die geistige Gährung erstreckt sich also in diesem Falle gleichmässig auf alle Zuckerarten, ohne dass sie die eine oder die andere vorzuziehen scheint.

Dieselbe Prüfungsmethode, auf Gemenge von reinem krystallisirtem Traubenzucker und umgewandeltem Zucker in bekantem Verhältnisse angewendet, beweist auch, dass die beiden Zuckerarten zu gleicher Zeit in die weinige Gährung übergehen; zu jeder Zeit der Gährung zersetzen sie sich in proportionalen Mengen und diese Quantitäten sind denen des Gemjsches gleich. 
Diese letzteren Resultate halte ich für genau, ungeachtet der Schwierigkeiten, welche sich der Bestätigung durch den Versuch entgegensteltten; sie stimmen aber keineswegs mit den Erscheinungen überein, die der umgervandelte Zucker unter denselben Bedingungen darbietet.

Die optische Analyse dieser Erscheinungen zeigt in der That, dass der erste zuckerhaltige Körper, der sich bei der Gährung des Fruchtzuckers zersetzt, ein in optischer Beziehung neutraler Zucker ist. Sie zeigt gleichfalls, dass der sich zuletzt zersetzende Theil eine Zuckerart mit starker Rotation nach Links ist, ferner, dass die zuckerartigen Körper, welche sich während dieser beiden Puncte zersetzen, gemengte Rotation haben und wahrscheinlich Gemenge in veränderlichem Verhältnisse von neutralem Zucker und stark nach Links drehendem Zucker sind.

Diese verschiedenen Zuckerarten oder Gemenge von Zucker haben also augenscheinlich hinsichtlich ihres Gährungsvermōgens verschiedene Eigenschaften, da die Reactionen, welche sie äussern, Erscheinungen sind, die sich immer in derselben Ordnung und mit einer Kraft darbieten, die keine auffallenden Verschiedenheiten zeigt.

Alle Arten des Traubensaftes und des Fruchtsaftes reifer Früchte (die Soubeiran Chulariose und Baudrimont Carpomel nannte) verhalten sich bei der geistigen Gāhrung dem umgewandelten Zucker analog und scheinen demnach mit ibm identisch $\mathrm{zu}$ sein *).

Alle hinreichend concentrirten Sirupe bewirken mit der Zeit einen reichlichen Niederschlag von deutlich unterschiedener Glucose mit Drehungsvermogen nach Rechts u. s. w.

Mehrere Gelehrte und unter ihnen namentlich Mitscherlich und Soubeiran reiheten diese Erscheinung der von Biot so vollständig bei den Trauben beobachteten an. Denn wenn sie

*) Setzt man Honig versuchsweise der geistigen Gährung aus, so findet eine Zunahme der Rotation nach Links statt, was eine Folge des Vorhandenseins ron Rohrzucker und dessen Umkehrung durch das Ferment ist. Von diesem Zeitpuncte an schreitet die Gährung des Honigs wie die des Fruchtzuckers vor sich. Nur gegen das Ende der Gāhrung geht die Rotation nach Rechts über, so dass der Honig, der sich zuletzt ersetzt, ein nach. Rechts ablenkender Zucker ist, der wohl noch einer besondern Prüfung bedarf. 
den Fruchtzucker und den umgewandelten Zucker als einfache Zucker betrachteten, so nahmen sie ferner an, dass die Krystallisationskraft diesen nach Links ablenkenden Zucker in nach Rechts ablenkende Glucose verwandle.

Folgende Beobachtungen sind dieser Theorie entgegen.

Wenn man einen Frucht - oder umgewandelten Zuckersirup sich selbst überlässt, nachdem man vorber genau ihre Hotation nach Links gemessen hat, und diese Rotation abermals misst; wenn die Sirupe so fest wie möglich geworden sind, so findet man, dass die Abweichung des vorsichtig wieder aufgelösten $\mathrm{Ge}-$ menges keine Veränderung erlitten hat. Nimmt man andererseits einen Theil eines solchen festen Sirups und trennt vermittelst Pressen den festen Theil von dem flüss,gen, so bemerkt man, dass der feste Theil aus nach Rechts ablenkendem Traubenzucker besteht, während der flüssige Theil, für sich untersucht, eine Zuckerart ist, die weit stärker nach Links ablenkt als der ursprüngliche Sirup; die Abweichung hat genau um so viel nach Links zugenommen, als die Abweichung in entgegengesetzter Richtung beträgt, welche durch die entfernte Glucose hervorgebracht wurde.

Auf welche Weise ich es auch anstellte, es gelang mir nicht; in dem umgewandelten Zucker eine solche Krystallisation hervorzubringen, dass das dem Gemenge eigenthümliche Rotationsvermögen sich verändert hätte.

In Folge dieser Thatsachen nun ist es unmöglich, anzunehmen, dass die Glucose, welche sich durch Krystallisation aus dem Fruchtzucker oder umgewandelten Zucker ausscheidet, nicht' schon fertig gebildet in den Säften vorhanden sei; nur erlaubt die elective Reaction, welche ich bei der Gährung dieser Zuckersäfte beobachtete, eben so wenig anzunehmen, dass die Glucose darin nur im einfachen Gemenge mit dem nach Links drehenden Zucker sei.

Präparirt man eine gährungsfähige Flüssigkeit mit umgewandeltem Zucker dergestalt, dass sie 0,10 p. C. reinen Alkohol zu erzeugen vermag, unterbricht man die Gährung, wenn sich 0;08 Alkohol entwickelt hat, und dampft darauf die Flüssigkeit ab, um den Alkohol abzuscheiden und den nicht zersetzten Zucker in Sirupsconsistenz zu brıngen, so erhält man einen stark gezuckerten Sirup, welcher bei gleicher Dichtigkeit und einer Temperatur 
von $14^{\circ}$ die Polarisationsebene nach Links und zwar dreimal stärker als der umgewandelte Zucker ablenkt.

Dieser Zucker verliert ein Viertheil von seinem Drehungsvermögen, wenn die Temperatur von $+14^{\circ}$ auf $+52^{\circ}$ steigt. Ueberlässt man denselben sich selbst, so krystallisirt er nicht; er erleidet sebr bald die weingeistige Gährung und verändert sich durch Sieden mit. Wasser und durch die Alkalien, weniger leicht jedoch als die krystallisirte Glucose.

Unter gewissen Bedingungen bildet er mit dem Kalke ein basisches, in Wasser wenig lösliches Salz; er löst sich in Alkohol von $90^{\circ}$ sehr leicht, doch mehr in der Wärme als in der Kälte. Man kann ihn leicht aus den Fruchtsăften auszieheı, wenn man dieselben mit Kalk, Blei oder Alkohol geeignet behandelt *).

Das Verhalten dieses Zuckers in der geistigen Gährung, welcher ihr in geringem Grade die durch die optischen Erscheinungen bemerkte elective Reaction ertheilt, gestattet nicht, denselben als einen einfachen zu betrachten; im Gegentheile glaube ich, dass dieser Zucker eben so wie der vorher beschriebene, neutrale Zucker aus zwei Zuckerarten von entgegengesetzter Rotation besteht, wovon eine Glucose ist.

Die Hilchsãuregährung orzeugt in den verschiedenen Zuckerarten den für die geistige Gährung beschriebenen ähnliche Reactionen.

Beobachlet man den Gang der. Milchsäuregährung der Zuckerarten und vergleicht die Säureentwickelung, welche man durch Sättigen misst, nit dem gleichzeitigen Verschwinden des Zuckerš, das man durch Abnahme der Rotation der gährenden Flüssigkeit bestimmt, so findet man bei der krystallisirten Glucose, dass gleiche Säuremengen, zu jedem Zeitpuncte der Găhrung erzeugt, gleichen Winkelabweichungen an Biot's Apparate, folglich auch

*) Durch ähnliche Behandlung kann zugleich die durch die elective Gährung angedeutete neutrale Verbindung isolirt werden; ich habe Grund, sie für identisch mit einer Zuckerart zu halten, welche zuerst Mits che rlich kennen lehrte. Ich fand, dass beide Zuckerarten mehrere Eigenschaften gemein haben; für jetzt erwähne ich nur eine der bemerkenswerthesten. Bei einer Temperatur von 13 bis $14^{\circ}$ sind beide nentral, während sie bei hơherer Temperatur. nach Links und bei niederer nach Rechts ablenken, 
auch gleichen Verbältnissen Glucose entsprechen. Es liegt darin ein neuer Beweis für die Einfachheit der krystallisirten Glucose.

Als ich umgewandelten oder einen āhnlichen Zucker der Milchsäuregährung unterwarf, machte ich folgende Beobachtungen.

Operirte ich mit einer zuckrigen Flüssigkeit, die unter den angegebenen Bedingungen 24 Sexagesimalgrade nach Links ablenkte, so bemerkte ich, dass, sobald die Milchsăuregährung anfing, die Drehung anstatt, wie man hätte vermuthen sollen, schwächer zu werden, zunimmt und sich im Verhältnısse zur erzeugten Säure steigert, bis sie endlich zu $36^{\circ} \mathrm{L}$. gekommen ist. Sie bleibt alsdann constant, und die Flüssigkeit hat aufgehort zu gähren; es ist eine Zuckerart mit starkem Drehungsvermögen nach Links entstanden, deren Eigenschaften den vorher beschriebenen völlig analog sind.

Diese Thatsachen lassen sich erklären, wenn man annimmt, dass die in der sauren Gährung des Fruchtzuckers verschwun-' dene Zuckerart, Glucose, Links ablenkend, sei, dass das Verbältniss dieser Glucose durch die Endablenkung minus der anfänglichen Ablenkung ausgedrückt werden kann, d. $h$.

$$
36^{\circ} \mathrm{L} .-24^{\circ} \mathrm{L} .=12^{\circ} \mathrm{R}^{*} \text { ). }
$$

Untersucht man die Menge der bei diesem Versuche entwickelten Săure, so findet man, dass dieselbe genau derjenigen entspricht, welche von einer Menge für sich gegohrener Glucose herrüht und unter lenselben Bedingungen des Versuchs $12^{\circ} \mathrm{R}$. ablenkt.

Aus diesen Thatsachen scheint mir hervorzugehen, dass die Milchsăuregährung, auf ähnliche Weise wie die Krystälisation, die Eigenschaft besitzt, umgewandelten Zucker von reiner Glucose abzuscheiden; nur scheint sie davon eine weit grössere Menge zu trennen.

Ein eben so bemerkenswerther Umstand meines Versuchs bestand in der Verzögerung der Milchsäuregährung, und ferner darin, dass der Links ablenkende Zucker unangetastet blieb.

Ich habe mir übrigens vorgenommen, diese Untersuchung noch fortzusetzen.

Die Eigenschaft des umgewandelten Zuckers and der von ihm abgeleiteten, neutralen oder Links ablenkenden Zuckerarten,

*) L. bedeutet Links und R. Rechts. 
424 Dabrunfat: Uebereine analytisohe Eigenschaft etc.

durch Temperaturveränderung regelmặssige und constante Veranderungen in ihrem Drehungsvermögen zu erleiden, liefert noch fernere Aufschlüsse über die vorliegende Frage.

Ich habe an einem andern Orte angegeben, dass der umgewandelte Zucker, indem sich seine Temperatur von $+14^{\circ}$ anf $+52^{\circ}$ steigert, die Hälfte seines Drehungsvermögens verliert. So würde eine Lösung desselben, die bei $14^{\circ} \mathrm{C}$. eine Winkelablenkung von $24^{\circ} \mathrm{C}$. zeigt, bei $52^{\circ}$ nur die Polarisationsebene um $12^{\circ}$ L. ablenken; der Saft hătte demnach durch diese Temperaturerhöhung $12^{\circ} \mathrm{L}$. Ablenkung verloren. Da nun derselbe Sirup bei der Milchsāuregährung, wie ich so eben sagte, eine Quantität $12^{\circ}$ R. ablenkender Glucose verliert, so steigert sich seine Drehung auf $36^{\circ} \mathrm{L}$. und der Sirup verliert in diesem Zustande, indem seine Temperatur von $14^{\circ}$ auf $52^{\circ}$ steigt, ein Drittheil seines Drehungsvermögens, d. h. $12^{\circ} \mathrm{L}$; d diese Verānderung ist demnach mit der bei der Milchsäuregährung stattfindenden identisch.

Diese Thatsachen scheinen mir ferner die aufgestellte Ansicht zu bestătigen, dass nämlich die Milchsäuregährung aus dem umgewandelten Zucker einen nach Rechts ablenkenden Zucker abscheidet, der, eben so wie die Glucose, wenig oder gar nicht in seinen Rotationseigenschaften durch den Temperaturwechsel verăndert wird, und dass die Substanz, welche im umgewandelten Zucker constante Verănderungen der Drehung mit der Temperatur erleidet, vollständig mit dieser Eigenschaft in der Verbindung bleibt, welche, meinen Versuchen zufolge, der Umwandlung in der Milchsāuregāhrung widersteht.

Die hauptsächlichsten Schlüsse, die aus vorstehender Abhandlung gezogen werden können, sind folgende:

1) Umgewandelter Zucker (sucre interverti) und die ihm ähnlichen Trauben - und Fruchtsirupe u. s. w. sind keine chemisch einfachen Zuckerarten; durch Krystallisation können sie nicht, wie man angegeben hat, in Glucose umgewandelt werden; dieser Process kann nur einen gewissen Antheil völlig charakterisirter Glucose ausscheiden, und das Rotationsvermögen der Nutterlauge nach Links vermehrt sich um das ganze entgegengesetz te Drehungsvermögen der abgeschiedenen Glucose.

2) Durch verschiedene Methoden lässt sich aus dem umgewandelten ein nicht krystallisirbarer Zucker abscheiden, der, unter andern charakteristischen Eigenschaften, mit einem Drehungsver- 
mögen nach links begabt ist. Letzteres ist ; bei einer Temperatur von $14^{\circ} \mathrm{C}$. das Dreifache des umgewandelten Zuckers bei derselben Temperatur. Dieser Zucker scheint noch nicht auf einen solchen Grad der Reinheit gebracht worden zu sein, um als einfach betrachtet werden $z \mathfrak{u}$ kōnnen.

3) Bei Untersuchung der geistigen Gährung des umgewandelten Zuckers vermittelst Destillation und dem optischen Apparate ergab sich, dass dieser Zucker kein einfacher ist. Der ziterst verschwindende Zucker ist in optischer Beziehung vollkommen neutral, der zuletzt verschwindende hingegen ein nicht krystallisirbarer Zucker mit starker Drehung nach Links.

4) Die Milchsāuregährung bewirkt in dem umgewandelten Zucker ebenfalls eine Art von Zersetzung; ihre anfängliche Einwirkung, anstatt sich, wie bei der geistigen Gährung, auf die neutrale Verbindung zu erstrecken, dehnt sich auf die Glucose aus, die schon fertig gebildet in dem umgewandelten Zucker zu bestehen scheint.

5) Unterwirft man einfache Zuckergemenge der geistigen Gährung, so scheint keine zerlegende Einwirkung stattzufinden. Die Umwandlungen finden aber so gut bei den einfachen Zuckergemengen wie bei den getrennten Zuckerarten statt; die Mengen der beiden Zuckerarten, welche sich zu allen Zeitpuncten der Gährung zersetzen, stehen zu einander stets in derselben Beziehung wie zu dem Gemenge.

\section{LV. \\ Ueber Glucose.}

Von

Dubrunfaut.

(Ann. de Chim. et de Phys. XXI, 178.)

D u mas belegte mit dem Namen Glucose verschiedene zuckerartige Producte von undeutlicher, warzenähnlicher Krystallisation. Dieser Körper ist identisch, gleichvièl ob er aus Früchten, aus dem Honig and dem diabetischen Harne herstammt, oder durch geeignete Behandlung des Stärkemehls oder der Holzfaser gewonnen worden ist. 